\title{
Clinical Studies of Chinese Medical Interventions for Premature Ovarian Failure
}

\author{
Lingling $\mathrm{Li}^{1, \mathrm{a}}$, Sujuan $\mathrm{Ma}^{1, \mathrm{~b}}$ and Weixia $\mathrm{Liu}^{1, \mathrm{c}^{*}}$ \\ ${ }^{1}$ The First Affiliated Hospital of Henan University of Traditional Chinese Medicine, 450000, Henan, P. R. \\ China \\ a289193189@qq.com, bmasujuan860126@126.com, cliwx0129@163.com \\ ${ }^{*}$ The corresponding author
}

Keywords: Premature ovarian failure; Chinese medicine treatment; Significant effect; Less adverse reactions

\begin{abstract}
Premature ovarian failure is a common clinical gynecological difficult disease, the incidence rate increased year by year, and its cause is unknown, treatment is more difficult. Western medicine use hormone replacement therapy, short-term effect is better, but long-term efficacy is poor, and long-term use of hormones may increase the risk of hormone-dependent tumors. Chinese medicine treatment methods varied premature ovarian failure, a significant effect, with few side effects. Chinese medicine treatment methods include experience side, pure Chinese medicine treatment, according to clinical syndromes dialectical therapy, the treatment of premature ovarian failure with a stable efficacy, adverse reactions, the role of the advantages of waiting, but there are many shortcomings, It is need for further in-depth study.
\end{abstract}

\section{Introduction}

Premature ovarian failure (premature ovarian failure POF) refers to low estrogen in women before the age of 40 due to ovarian failure occurring gonadotropin and high status. This year the incidence is increasing, thereby causing irregular menstruation, infertility, loss of libido, menopausal syndrome, and due to lower estrogen levels caused by osteoporosis, lipid metabolism disorders and the onset of cardiovascular disease, a serious threat to women's health and quality of life, increasingly become a hot research. Western medicine treatment of the disease long-term effects are poor, big side effects, improving the quality of life of patients has a significant limitations, and Chinese medicine as "holistic concept" and "syndrome" features, that "the balance of yin and yang", a significant effect, toxic side effects, does not increase breast cancer, endometrial cancer incidence, and easy for patients to accept, in the POF treatment play a unique advantage [1]. For premature ovarian failure (POF) treatment, the use of modern western medicine therapy cycle can achieve the establishment of the menstrual cycle, ovulation, reducing clinical symptoms and side effects, but still exist menstruation, poor egg quality, pregnancy rate, patients receiving poor, a lot of problems after the withdrawal symptoms recurrence. In recent years, the introduction of Chinese medicine in the treatment interventions, the use of traditional Chinese medicine multi-system, multi-link the overall regulatory role, to restore ovarian function, reduce the side effects of hormone therapy, reduce relapse rate after stopping the mouth. Now reviewed on Chinese medicine to the POF pathogenesis and Differential Treatment Strategies.

Diagnostic Criteria. Women in the age of 40, and there are 2 or more serum FSH, 40U / L, E2 $<73.2 \mathrm{ng} / \mathrm{L}$, at least one month interval.

Clinical Manifestations. Menstrual changes: amenorrhea is the main clinical manifestations of POF. Before the onset of puberty showed primary amenorrhea, secondary sexual characteristics undeveloped; after the onset of puberty is manifested in secondary amenorrhea, menstrual close before the age of 40, usually secondary sexual characteristics. POF before the onset of menstrual changes in the form of more diverse, about $50 \%$ of patients with menstrual blood thinning hair or Palace as the first symptom; $25 \%$ of patients are suddenly amenorrhea. Or due to an abnormal chromosome variability will affect the formation of the egg and ovarian development, so there are chromosomal 
defects earlier onset of POF, and even failed to reach puberty, which showed primary amenorrhea most POF patients with ovarian function decline occurring process sudden and irreversible, few patients that this process will continue for some time, is equivalent to the natural menopause perimenopause [2].

Estrogen deficiency performance: Due to declining ovarian function, estrogen levels decline, POF occurs in patients with perimenopausal syndrome, such as vasomotor symptoms: hot flashes, sweating, etc; genitourinary tract symptoms: dysuria, urgency, urinary frequency, difficulty urinating, spasm genital itching, vaginal burning sensation, vaginal dryness and dyspareunia, etc; and neuropsychiatric symptoms: depression, anxiety, insomnia, memory loss and so on. These symptoms in patients with primary amenorrhea POF is relatively rare.

\section{Chinese Medicine to Improve Theoretical Basis of Premature Ovarian Failure}

Clinical manifestations of premature ovarian failure from the point of view, can be attributed to Chinese medicine, "Xueku", "amenorrhea", "infertility" category. Its most fundamental pathogenesis of kidney essence deficiency, leading to functional disorder, a series of physical and psychological symptoms of abnormal "heart brain - uterus axis - kidney." Studies have shown that kidney medicine have estrogen-like effects, can regulate the hypothalamic - pituitary - uterus axis function, promote ovarian follicle, TCM treatment to kidney, combined with nourishing blood, tone the liver and spleen and other methods of treatment, from adjust the patient as a whole and the whole yin and yang qi to improve local ovarian function, and its effectiveness has been confirmed by clinical and experimental [3].

Blood Stagnation Is the Direct Causative Factors of This Disease. By the water barrier, and less than nothing less than the more than two kinds, namely by more than blood stagnation, blood does not charge while the pulse Road; namely Xueku is insufficient, in order of passive or Haoshang excessive.

The organ Dysfunction Is at the Root of This Disease. Kidney and premature ovarian failure: insufficient kidney, kidney essence depletion of this disease is the basic pathogenesis, either ancient or modern physicians this has a high degree of recognition. Kidney is the innate root strength, the main Tibetan essence, the body's growth, development and reproduction of fundamental and essence and the source of the blood, passing directly to the uterus, Breeds provide the material basis.

Liver and premature ovarian failure: Liver dispersion, Secretary sea of blood, hi be reached and evil, depression. With the liver and red Rendu contact and participation menstrual cycle, menstrual period and by the amount of adjusting three veins on the meridian. Liver failure catharsis, qi stagnation, Yu-fire a long, fiery burns Yinxue not nourish any red bet uterus, a sea of blood and burning due to hot fried dry, no blood can leak; or catharsis disorders, negative air machine Chong and Ren blocked, blood is difficult to bet the uterus, resulting in early menopause.

Heart and premature ovarian failure: Heart monarch of the officer who dominate one of blood and viscera, cellular connections, cellular network is distributed in the extracellular intrauterine context for the delivery of blood to uterus completes its exclusion menstruation and the fetus functions.

Spleen and premature ovarian failure: the spleen is acquired, the source of qi and blood, spleen controlling blood in the air and gas main lifting the main transport, healthy and vigorous temper, the blood biochemical active, unifying entitled, normal menstruation, and vice versa temper weakness, lack of qi and blood source, empty sea of blood, no blood can be under, menstrual close.

\section{Chinese Medicine to Improve the POF Dialectical Theory of Governance}

Ancient physicians premature ovarian failure etiology and pathogenesis of understanding is not uniform, but most physicians believe that the key to this disease is kidney [4]. Kidney is the innate root strength, the primary menstrual and reproductive No two kidney Sheng, refined gas is not sufficient, the impact of "Kidney - menstruation - Chong and Ren - the uterus," the function, it is the disease of the kidney of pathogenic this. Treatment of multiple proceed from the kidney. 
Kidney Tiaogan. The main reproductive kidney essence, menstruation is fundamental; liver blood dispersion, B Kuei homologous liver and kidney coordinated, predictable menstrual disorders and kidney February closely by the liver.

Kidney and Spleen. The birth of the kidney, spleen and stomach is acquired, the kidney and spleen are mutually funded and interdependent. Kidney is the growth and reproduction of women's basic physical and functional, spleen is the source of qi and blood, kidney essence gas Chong Sheng, stomach healthy and vigorous, Chong Sheng then pass any blood strong, through pregnancy, birth, breast normal two-kidney spleen prone to loss by, with, tires, production of various diseases [5]. Insufficient kidney, kidney essence depletion, which is fundamental Benbingfasheng; liver qi stagnation, catharsis disorders, resulting in poor blood, blood addiction addiction resistance, falling blood cell pulse caused by addiction, Chong and Ren dystrophy, eventually leading to ovarian premature aging.

\section{Chinese Medicine Intervention Strategies}

Because the main pathological basis for the POF pathogenesis of kidney, so when the first treatment to kidney first. Chinese medicine, kidney is the innate, the source of menstruation, is the reproduction of human growth and development fundamentally solid kidney essence and fit, sealing possession by the water level is as usual; on the contrary, such as virtual kidney failure, renal system, many aspects of the overall regulation, which itself is not a hormone, but has a significant ability to regulate, in particular, to improve ovarian response to gonadotropin and ovarian hormone receptor content. Thus, the main treatment of this disease to kidney, liver and kidney that is used Guishen Pill, medicinal raw Rehmannia, mountain dogwood, wolfberry, Chinese yam, Poria, angelica, Eucommia, dodder, Achyranthes, Epimedium beans, fried altogether, fried Cork, Placenta; kidney Liver Depression with Liu Wei Di Huang pill Xiaoyaosan, medicinal Rehmannia, Chinese yam, dogwood, Dan, Alisma, Poria, angelica, white peony root, Radix, Atractylodes, Toosendan, Epimedium, Placenta, Cyathula; kidneys do not pay type with Guishen pill berberine gelatin decoction, medicinal habitat, dogwood, Chinese yam, Poria, medlar, Epimedium, berberine , fried Zao Ren, Polygala, Schisandra, donkey-hide gelatin, white peony root. Said one prescription daily, once every 3 months for a course of treatment.

According to indulge in drugs and kidney, deficiency, kidney yin and yang and choose Zuoguiwan Youguiwan, Immortals soup and other drugs. Kidney essence, the essence energy and blood, refined homologous blood, become the material basis of menstruation, is pregnant, a necessary condition for fertility, so the treatment of this disease, we must first Bushentianjing, optional add Placenta dialectical basis, Huang Jing Polygonum, stone solutions and other drugs, can play a multiplier effect. Because kidney yin and yang of the house, dirty water and fire, the fundamental internal organs, and the "phase shift of the five internal organs, poor kidney will", so kidney yin and yang imbalance, can affect other organs, and other organs lesions, a long time is bound to affect the kidneys. Pathogenesis of this disease is a kidney-based, yin and yang imbalance, blood loss, multiple organ involvement is the direct cause of its diverse clinical manifestations [6]. It should take into account the treatment of other organs. And wherein the liver qi, blood of addiction is the key. Zheng Jian, such as clinical breast swelling, rib, pain, irritability, insomnia, abdominal distension, should Zhuojia Radix, white peony root, product shell, Cyperus, turmeric, Dan, Albizia and other liver qi, cooling sedative goods. Duration of the course of time, gradually Health addiction blood, Zheng Jian lower abdomen tingling, insomnia, dark tongue, tongue tip point of addiction, etc., may be selected as appropriate Salviae suberectstem Motherwort, Achyranthes blood to regulate menstruation and other drugs. In short, the treatment of this disease and when yin and yang tone, scattered in income, firmness and flexibility, Shigemi Han wood, water and fire that is economic, before they reach the balance of yin and yang. "Yin Pingyang secret, the spirit of governance", the illness can be expected.

Treatment should be phased conditioning, disease and syndrome and rule. Emmeniopathy treatment should conform to the physiological menstruation, medication cycle segment, this view has been 
recognized by many colleagues and Chinese medicine has been used clinically, the disease is no exception. In the liver and kidney, blood to regulate menstruation. On the basis of the rule, depending on the menstrual cycle phase of the different physiological and pathological characteristics, homeopathic medication, can often play a multiplier effect, as follows: After the uterus emptiness, nourishing fill in essence to main, side by $\mathrm{Wu}$ ziyanzongwan, $\mathrm{Si}$ wutang, by liquid decoction; premenstrual in kidney yin on the basis of kidney yang, in order to encourage and maintain the luteal function of the mouth, the drug in the above-mentioned parties on the basis of gabba citrifolia , Epimedium, Curculigo, meat from Chengdu, injustice silk son, teasel, mistletoe, Antlers, Placenta, etc; ovulation between periods of modern medicine, is the heavy overcast sun process Expelling tune up the yin and yang, based on the blood of addiction to promote ovum goods, often with Wulingzhi, artillery Shan Jia, safflower, Zeeland and the like.

\section{Summary}

Premature ovarian failure is a common gynecological diseases, gynecological also refractory disease in recent years, Chinese medicine in the treatment of premature ovarian failure is more and more widely, and has made some progress in research. Many physicians according to clinical practice, the use of diagnosis and treatment, after-party prescription, stage of governance, integrative medicine, acupuncture and other methods in the treatment of premature ovarian failure and achieved good effect forty-two Chinese medicine treatment of hormone replacement therapy compared to Western medicine alone, with efficacy and stability, fewer adverse reactions, the role of the advantages of waiting, has a broad development prospects.

\section{References}

[1] Xie Xing, Gou Wenli. Obstetrics and Gynecology [M]. Beijing: People's Medical Publishing House, 2013: 354.

[2] Chen Li, $\mathrm{Xu}$ Lin. Premature ovarian failure, the cause of science and research progress in the field of animal model of progress in Obstetrics and Gynecology, 2008.17 (8): 627-632.

[3] Situ yi. Integrative Medicine Obstetrics and Gynecology [M]. Beijing: Beijing Science and Technology Press, 2003: 155.

[4] Zhang Lili. Premature ovarian failure [J] Chinese Journal of Practical Traditional Chinese and Western, 2004, 4 (17): 139.

[5] Yao Shian. TCM treatment of premature ovarian failure should pay attention to what [J]. Traditional Chinese Medicine, 1998, 39 (5): 313.

[6] Cai Huapu, Xu Jing, Meng Qingling. Treatment of premature ovarian failure status of Chinese medicine [J] Shandong Traditional Chinese Medicine, 2004, 23 (9): 575. 